\title{
Anticancer and Anti-inflammatory Effect of Diosmin against Dalton Ascitic Lymphoma Induced Leukemia
} Xiangmei Yao ${ }^{1}$, Xuezhong Gu${ }^{1}$, Song $\mathrm{Jin}^{2}$, Keqian Shi ${ }^{1}$, Xiaoli Gao², Qi Wang ${ }^{1}$, Jie Zhao', Haixi Zhang ${ }^{1}$, and Xun Lai ${ }^{3 *}$

${ }^{1}$ Department of Hematology, The First People's Hospital of Yunnan Province, Kunming, Yunnan, 650032, CHINA

${ }^{2}$ Department of Rheumatism Immunology, The First People's Hospital of Yunnan Province, Kunming, Yunnan, 650032, CHINA

${ }^{3}$ Department of Hematology, Yunnan Cancer Hospital, Kunming, Yunnan, 650118, CHINA

\begin{abstract}
Cancer is the world's biggest health problem and cancer-induced mortality happened all over the planet after the heart disease. The present study was to scrutinize the anti-leukemia effect of diosmin against Dalton Ascitic Lymphoma (DAL) induced leukemia in mice. DAL cell was used for induction the solid tumor. Body weight, life spans, tumor volume and mean survival time was estimated. Antioxidant, biochemical and pro-inflammatory cytokines were estimated. Diosmin showed the cell viability effect at dose dependent manner against the both cell lines. DAL induced solid tumor mice showed the decreased body weight, mean survival days, non viable cell count and increased the tumor volume, viable cell count and diosmin significantly $(p<0.001)$ reverse the effect of DAL. Diosmin significantly $(p<0.001)$ altered the hematological, differential leukocytes, antioxidant, biochemical, pro-inflammatory cytokines at dose dependently. Collectively, we can say that diosmin might alter the DAL induced abnormality via antioxidant and anti-inflammatory effects.
\end{abstract}

Key words: diosmin, anti-leukemia, Dalton Ascitic Lymphoma, anti-inflammatory, antioxidant

\section{Introduction}

Cancer is the most dreadful disease worldwide and $2^{\text {nd }}$ largest disease occur death worldwide after the heart disease. According to previous reports, the total number of new cancer cases reported in 2013 was 1,660,290 and 580,350 cancer deaths reported in the United States only in $2013^{1,2)}$. Approximately 1,762,450 new cancer patients report and about 606,880 cancer-related deaths have occurred from cancer in the United States in 2019. The number of cancer cases increased day by day in all around the world. The treatment of cancer is one of the challenges for the health issues ${ }^{3)}$. Chemotherapy is commonly used to treat the cancer. But the chemotherapy having the limitation due to side effects ${ }^{4}$. Reported also suggest that the anticancer drugs not only shows the effect on the cancerous cells but also show the effect on the normal cells ${ }^{5)}$. Nature is the god gift that provides the valuable source of the therapeutic compounds as enormous chemical diversity is found in the millions of species of animals, plants, microorganisms and marine organisms ${ }^{6)}$. Cancer chemoprevention is defined as a cancer control strategy through the administration of natural or synthetic compounds to inhibit or reduce the carcinogenic process ${ }^{7)}$. Originally derived from medicinal plants, plant-based medicines actually play a key role in the cure of cancer and secondary metabolites and their derivatives widely used to fight cancer.

Diosmin (natural glycoside flavonoids) is obtained via dehydrogenation of hesperidin ${ }^{8,9)}$. Diosmin is found in the abundant quantity in the pericarp of variety of citrus fruits $^{10)}$. It exhibited the various biological activities, such as anti-hyperglycemic, anti-inflammatory, antimutagenic and antioxidant effects against the different animal models ${ }^{8-14}$. Pharmacokinetic studies have shown that diosmin is quickly transformed via intestinal and speedily dispersed throughout the whole body with a plasma half-life(23-44 g). According to my current knowledge, till date anti-leukemia effect of not estimated. In the current experimental study, we make attempt to scrutinize the anti-leukemia effect of diosmin and explore the possible mechanism of action.

\footnotetext{
*Correspondence to: Xun Lai, Department of Hematology, Yunnan Cancer Hospital, Kunming, Yunnan, 650118, CHINA

E-mail: xykyxm@sina.com

Accepted January 31, 2021 (received for review January 16, 2021)

Journal of Oleo Science ISSN 1345-8957 print / ISSN 1347-3352 online

http://www.jstage.jst.go.jp/browse/jos/ http://mc.manusriptcentral.com/jjocs
} 


\section{Material and Method}

\subsection{Cell culture}

Blood cancer cell lines such as K-562 (CCL-243) and Kasumi-1 (CRL-2724) were obtained from the American Forms Culture Collection U.S.A. for cell proliferation, utilizing Dulbecco's medium, for the in vitro experimental study. Briefly, DMEM supplemented with 10\% fetal bovine serum to grow the cell lines. The cells were propagated in RPMI-1640 supplemented with FBS (20\%) and maintained the cells in room temperature $\left(37^{\circ} \mathrm{C}\right)$, humidity $((95 \%)$ and $\mathrm{CO}_{2}(5 \%)$.

\subsection{Cell viability assay}

For the estimation of cell viability effect of diosmin, the cells were inoculated at 96 well plates and cells suspension $\left(1 \times 10^{5}\right.$ cells $\left./ \mathrm{mL}\right)$ at $100 \mathrm{~mL}$ per cell and different dilution of Diosmin were treated to the cells. After words, the plates were inoculated with $5 \% \mathrm{CO}_{2}$ at temperature $37^{\circ} \mathrm{C}$ for next $72 \mathrm{hr}$. The cell suspension was then successfully removed and equal trypan volume was mixed and incubated again at $37^{\circ} \mathrm{C}$ for next $5 \mathrm{~min}$. For counting the non-viable cell (stained) and viable cells (unstained) were recorded.

Cell viability assay (V) was estimated via using the following formula:

$$
V=\frac{C v}{C t} \times 100
$$

Where $\mathrm{Ct}$ is the total number of cell and $\mathrm{Cv}$ is the viable cell number.

\subsection{Experimental animal}

Balb Mice weighing roughly 20-30 g were procured from the Institution's main animal house facility. The mice were randomly divided into the various groups, and each group contains 12 mice, and the mice acclimatized for the next 7 days before the experimental protocol was started. The mice have been kept in the separate room and room temperature should be $24 \pm 2^{\circ} \mathrm{C}$ and keep the light cycle of 12 hours dark/light. The mice were received the standard rodent diet and water ad libitum.

\subsection{Tumor cells}

Dalton Ascites Lymphoma(DAL) cells were collected from the Shanghai Institute. The DAL cells were used for the in vivo model for animals. Intraperitoneal injection of $\operatorname{DAL}\left(10^{6}\right.$ cells $)$ into a new mouse after every 10 days $^{7)}$. For the experimental procedure, the DAL cells were obtained from the donor mice (on day 9 after the initial DAL injection) and suspended in the sterile saline, and trypan blue exclusion was used to estimate concentration and viability.

\subsection{Experimental protocol}

The mice were divided into groups below. Gp-I: normal control and treated with saline; Gp-II: intraperitoneal treated with DAL only; GP-III: intraperitoneal treated with
DAL and received the Diosmin (2.5 mg/kg); GP-IV: intraperitoneal treated with DAL and received the Diosmin (5 $\mathrm{mg} / \mathrm{kg})$; Gp-V: intraperitoneal treated with DAL and received the Diosmin $(10 \mathrm{mg} / \mathrm{kg})$ and Gp-VI: intraperitoneal treated with DAL and received the methotrexate (3 mg/ $\mathrm{kg}$ ), respectively. The mice were received the above mention treatment for next 10 days post DAL intraperitoneal injection. Body weight was measured at regular intervals for all group of mice ${ }^{15,16)}$.

On days 10 and 15 , mice subsets $(n=6)$ from each group were euthanized through cervical dislocation, and estimated ascites fluid. For the estimation of Hematological parameters have been determined, such as white blood cells (WBC), red blood cells (RBC) and hemoglobin ( $\mathrm{Hb})$. The retro orbital plexus puncturing for collecting the blood samples ${ }^{16-18)}$.

After that the remaining mice(3) of all group used for the estimation of lifespan.

For the estimation of Mean survival Time (MST), the MST was compared between the treated and DAL control group mice via using the following formula:

$$
\begin{aligned}
& \text { Mean Survival Time }(M S T)= \\
& \qquad \frac{\text { Day of First death }+ \text { Day of last death }}{2}
\end{aligned}
$$

And the percentage of lifespan was estimated via using the following formula:

$$
\begin{aligned}
& \text { Lifespan }(\text { ILS \% })= \\
& \frac{(\text { MST of treated group })-(\text { MST of DAL control group })}{\text { MST of DAL control group }}
\end{aligned}
$$

\subsection{Hepatic parameters}

Hepatic parameters such as SGOT, SGPT and ALP were estimated via using the ELISA kits via using the manufacture instruction (Abcam diagnostic).

\subsection{Biochemical parameters}

Biochemical parameters such as GGT and protein were estimated via using the previous reported method with minor modification $^{16,19)}$.

\subsection{Antioxidant parameters}

The antioxidant parameters such as CAT, TBARS, GSH, and SOD were calculated with minor modification using the previously reported method. The level of vitamin $\mathrm{C}$ and vitamin $\mathrm{E}$ also was calculated using the previously reported method with minor modifications ${ }^{16,19)}$.

\subsection{Estimation of pro-inflammatory cytokines}

Pro-inflammatory cytokines such as IL-6, TNF- $\alpha$ and IL- $1 \beta$ via using the instruction of ELISA kits manufactures (Abcam diagnostic). 


\section{Result}

\subsection{Diosmin effect on cell viability}

For the estimation of cell viability effect, Trypan blue model was commonly used. For the cell viability effect, diosmin treated with the both leukemia cells at different concentration. Figure 1 showed the cell viability effect of diosmin on the leukemia cell lines like Kusumi-1 and k-562. Figure 1 showed the dose dependently response on the cell viability of diosmin at different time intervals.

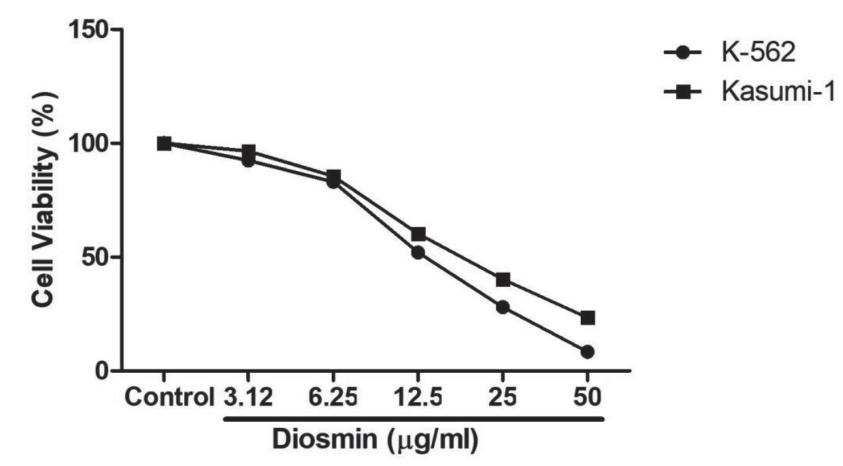

Fig. 1 The cell viability effect of diosmin on the both cell lines.

\subsection{Diosmin effect on tumor volume, body weight and mean survival}

The effect of diosmin on body weight, tumor volume, mean survival time on the solid tumor mice induced by DAL was shown in Table 1. DAL induced control mice showed reduced body weight, non-visible cells and increased tumor volume, average survival time, and visible cells. On the other hand, DAL induced solid tumor mice treated with diosmin significantly $(p<0.001)$ increased the body weight, non visible cells and reduced the tumor volume, mean survival time, visible cells at dose dependently manner.

\subsection{Effect of diosmin on hematological parameter on the DAL induced solid tumor}

During the expansion of tumor, alteration the hematological parameters is the common feature. A similar result was observed in the DAL induced control group mice. DAL induced group mice showed the reduced level of hemoglobin, RBC and increased level of WBC, polymorphs as compared the normal control. DAL-induced control group mice treated with diosmin displayed increased levels of hemoglobin, RBC and decreased WBC levels, polymorphs as dose-dependent (Fig. 2).

Table 1 showed the effect of diosmin on the body weight, tumor volume, mean survival time, viable cell count and nonviable cell count in DAL induced leukemia in mice.

\begin{tabular}{clccccc}
\hline S. No & \multicolumn{1}{c}{ Groups } & $\begin{array}{c}\text { \% Decrease body } \\
\text { weight }(\mathrm{g})\end{array}$ & $\begin{array}{c}\text { Tumor volume } \\
(\mathrm{mL})\end{array}$ & $\begin{array}{c}\text { Mean Survival } \\
\text { time (days) }\end{array}$ & $\begin{array}{c}\text { Viable cell count } \\
\text { count }\end{array}$ & $\begin{array}{c}\text { Non-viable cell } \\
\text { count }\end{array}$ \\
\hline 1 & DAL Control & $10.34 \pm 0.39$ & $5.43 \pm 0.46$ & $19.34 \pm 1.54$ & $10.78 \pm 0.38$ & $0.74 \pm 0.21$ \\
2 & DAL + Diosmin $(2.5 \mathrm{mg} / \mathrm{kg})$ & $8.73 \pm 0.37 * *$ & $3.47 \pm 0.40^{* *}$ & $22.34 \pm 1.89^{*}$ & $5.12 \pm 0.31^{* *}$ & $1.93 \pm 0.53^{* *}$ \\
3 & DAL + Diosmin $(5 \mathrm{mg} / \mathrm{kg})$ & $6.11 \pm 0.47 * * *$ & $2.78 \pm 0.34 * * *$ & $29.83 \pm 2.11 * * *$ & $3.32 \pm 0.29 * * *$ & $2.79 \pm 0.68 * * *$ \\
4 & DAL + Diosmin $(10 \mathrm{mg} / \mathrm{kg})$ & $3.21 \pm 0.23^{* * * *}$ & $1.34 \pm 0.21 * * *$ & $42.05 \pm 2.34 * * *$ & $1.04 \pm 0.21^{* * *}$ & $5.12 \pm 0.93 * * *$ \\
5 & DAL + methotrexate $(3 \mathrm{mg} / \mathrm{kg})$ & $3.47 \pm 0.11^{* * *}$ & $1.49 \pm 0.28^{* * *}$ & $41.45 \pm 2.87 * * *$ & $1.14 \pm 0.15^{* * *}$ & $5.01 \pm 0.87^{* * *}$ \\
\hline
\end{tabular}

Dennett's statical method was used for scrutinize the statistically significance $\left({ }^{*} p<0.05,{ }^{*} p<0.01\right.$ and $\left.{ }^{* * *} p<0.001\right)$.

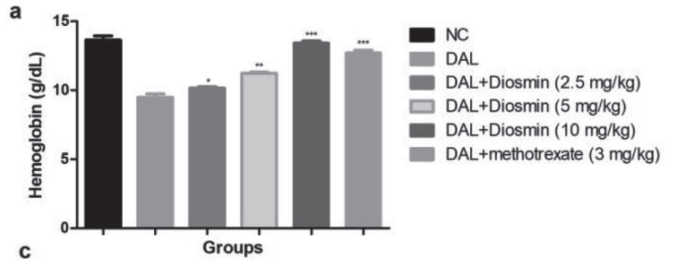

c

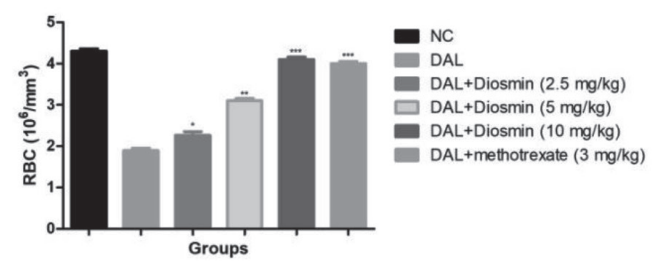

b
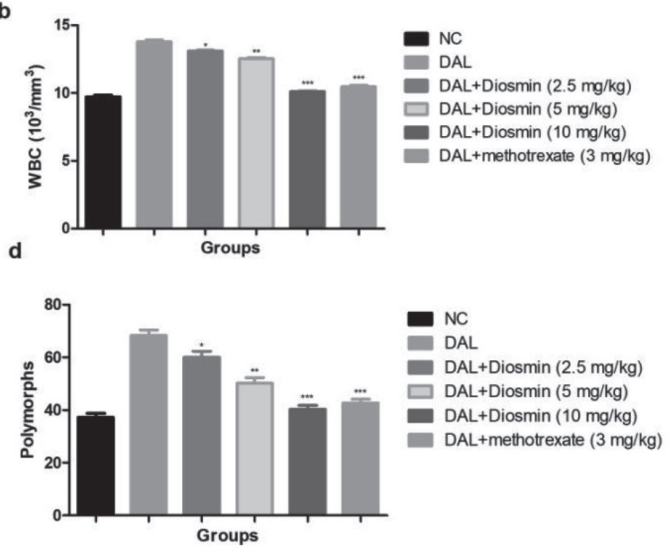

Fig. 2 The effect of diosmin on the hematological parameter on DAL induced solid tumor. a: hemoglobin, b: WBC, c: RBC and d: polymorphs. Dennett's statical method was used for scrutinize the statistically significance $\left({ }^{*} p<0.05, * * p<\right.$ 0.01 and $* * * p<0.001)$. 


\subsection{Effect of Diosmin on differential lymphocytes param- eter on the DAL induced solid tumor}

During the cancer, differential lymphocytes parameters altered due to expansion of cancerous cells. Figure 3 showed the effect of diosmin and DAL induced mice solid tumor mice. DAL induced group mice showed boosted level of neutrophils, macrophages and reduced level of lymphocytes and Concentration-dependent diosmin significantly $(p<0.001)$ increased the lymphocyte rates and decreased neutrophil, macrophage levels.

\subsection{Effect of Diosmin on antioxidant parameter on the DAL induced solid tumor}

During the cancer, the endogenous antioxidant parameters altered due to expansion of cancerous cells. A similar result was obtained in current experimental study. DAL mediated group mice displayed higher levels of TBARS and lower levels of CAT, SOD, GSH as comparison to NC. On the other hands, diosmin received mice exhibited the reduced level of TBARS and increased level of CAT, SOD, GSH as compared to DAL induced solid tumor mice(Fig. 4).

\subsection{Effect of Diosmin on hepatic parameter on the DAL induced solid tumor}

DAL induced group mice exhibited increased hepatic parameter levels such as SGPT, SGOT, and ALP compared to normal group mice. Diosmin significantly $(p<0.001)$ decreased the hepatic parameter level as comparison to the group mice induced by DAL. Diosmin $(10 \mathrm{mg} / \mathrm{kg})$ dose treated group mice showed a decrease in hepatic parameter levels and reached near the normal control group mice (Fig. 5).
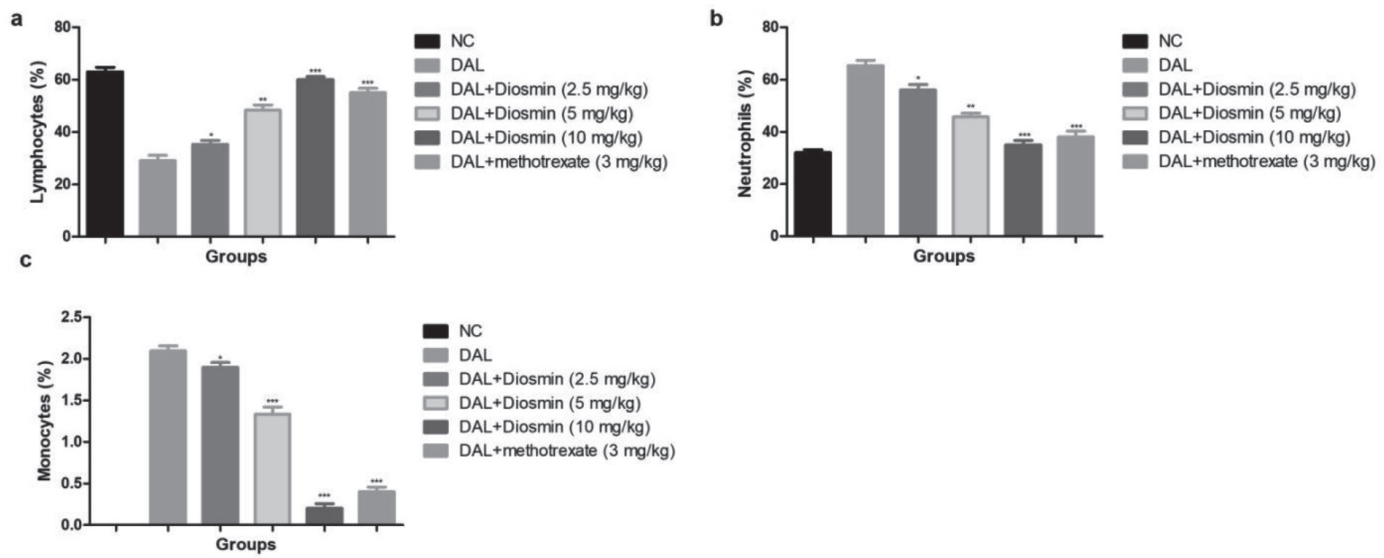

Fig. 3 The effect of diosmin on the differential leucocytes parameters on DAL induced solid tumor. a: lymphocytes, b: neutrophils and c: monocytes. Dennett's statical method was used for scrutinize the statistically significance $\left({ }^{*} p<\right.$ $0.05, * * p<0.01$ and $* * * p<0.001)$.
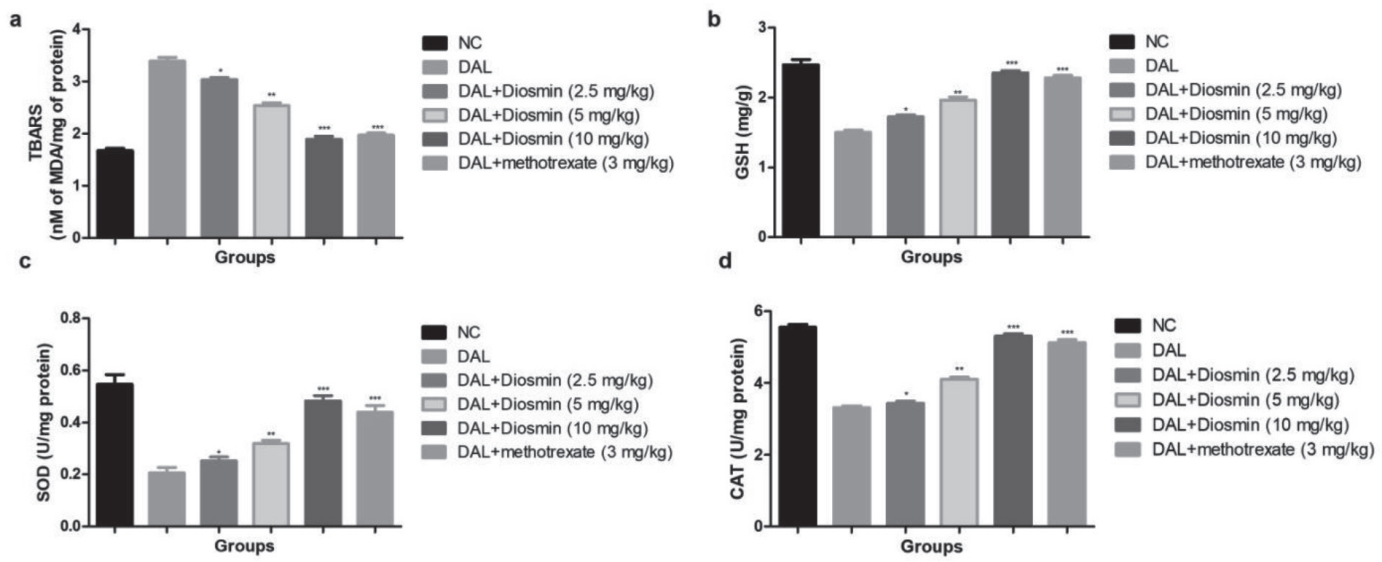

Fig. 4 The effect of diosmin on the antioxidant parameter on DAL induced solid tumor. a: TBARS, b: GSH, c: SOD and d: GSH. Dennett's statical method was used for scrutinize the statistically significance $\left({ }^{*} p<0.05,{ }^{* *} p<0.01\right.$ and ${ }^{* * *} p<$ $0.001)$. 


\subsection{Effect of diosmin on biochemical parameter on the DAL induced solid tumor}

Biochemical parameters such as GGT and protein level were altered during the cancer. A similar result was obtained in current experimental study. DAL induced solid tumor mice displayed elevated GGT levels and decreased total protein levels relative to normal control. Concentration dependent treatment of diosmin significantly $(p<$ 0.001 ) reduced the level of GGT and increased the level of protein (Fig. 6).

\subsection{Diosmin effect on DAL-induced solid tumor at vitamin level}

DAL induced solid tumor mice showed the reduced level of vitamin $\mathrm{C}$ and increased level of vitamin $\mathrm{E}$ as compared to normal control mice and dose dependent treatment of diosmin significantly increased the level of vitamin $\mathrm{C}$ and reduced the level of vitamin E (Fig. 7).

\subsection{Effect of diosmin on pro-inflammatory cytokines on the DAL induced solid tumor}

Normal control group mice showed the normal level of pro-inflammatory cytokines. On the other hand, solid tumor mice induced by DAL exhibited increased levels of pro-inflammatory cytokines such as TNF- $\alpha$, IL- $1 \beta$ and IL-6 compared to mice in the NC group. Dose-dependent diosmin treatment $(p<0.001)$ significantly reduced the level of pro-inflammatory cytokines such as TNF- $\alpha$, IL-1 $\beta$ and IL- 6 compared with solid tumor mice induced by DAL (Fig. 8).
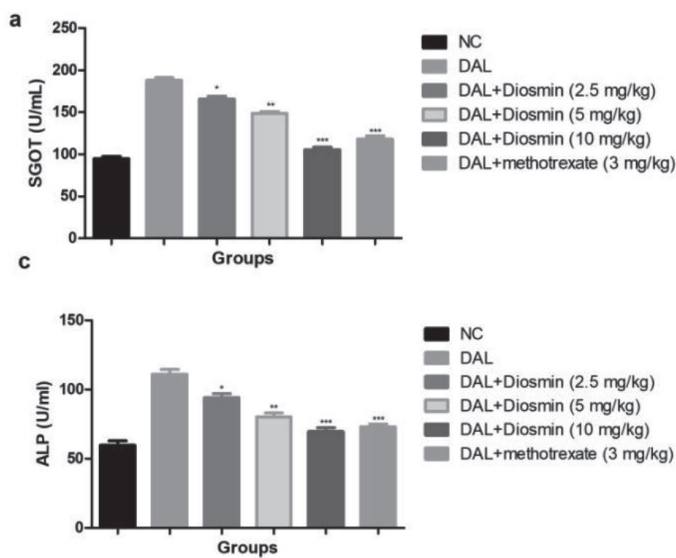

b

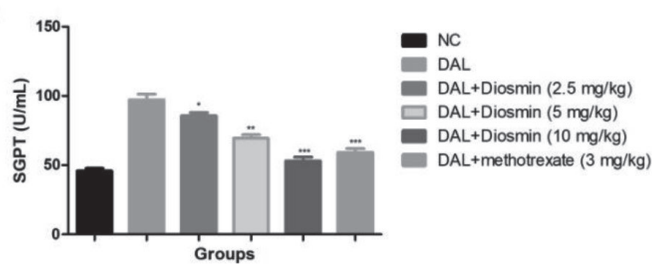

Fig. 5 The effect of diosmin on the hepatic parameters on DAL induced solid tumor. a: SGOT, b: SGPT and c: ALP. Dennett's statical method was used for scrutinize the statistically significance $\left({ }^{*} p<0.05, * * p<0.01\right.$ and $\left.{ }^{* * *} p<0.001\right)$.

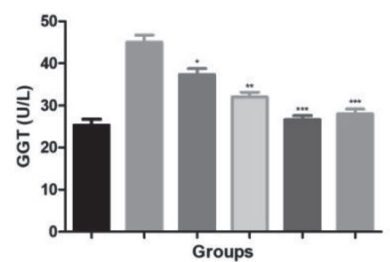

b

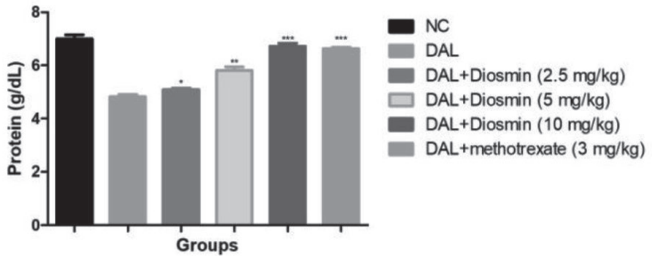

Fig. 6 The effect of diosmin on the biochemical parameters on DAL induced solid tumor. a: GGT and b: protein. Dennett's statical method was used for scrutinize the statistically significance $\left({ }^{*} p<0.05,{ }^{* *} p<0.01\right.$ and $\left.{ }^{* * *} p<0.001\right)$.

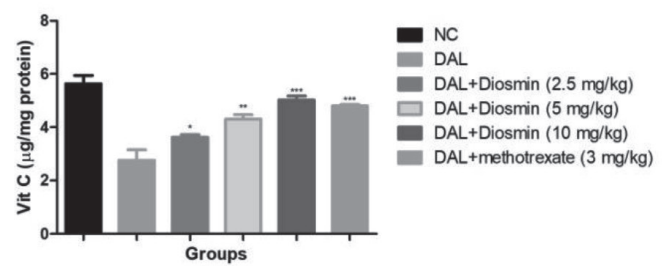

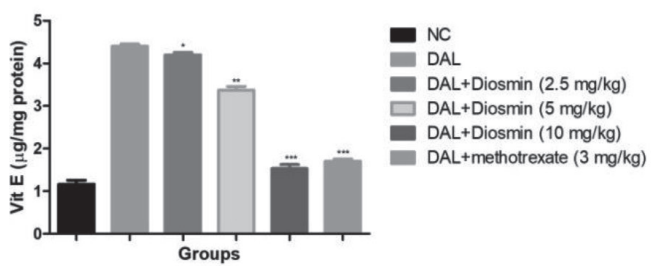

Fig. 7 The effect of diosmin on the vitamin parameters on DAL induced solid tumor. a: vitamin C and b: vitamin E. Dennett's statical method was used for scrutinize the statistically significance $\left({ }^{*} p<0.05,{ }^{* *} p<0.01\right.$ and $\left.{ }^{* * *} p<0.001\right)$. 
a
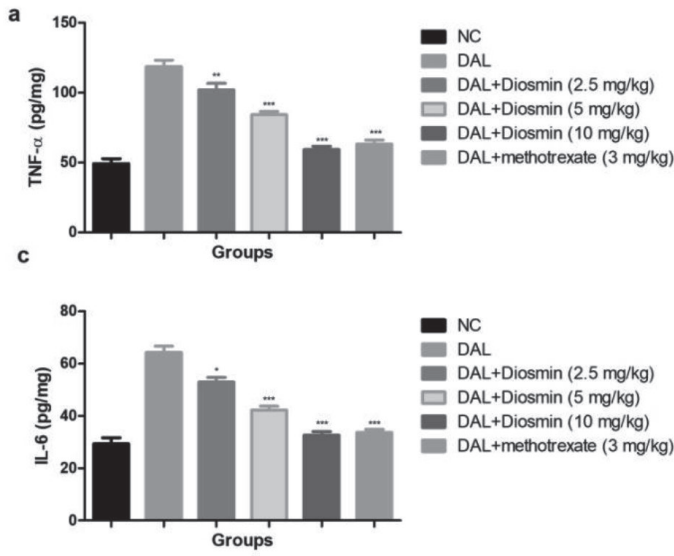

b

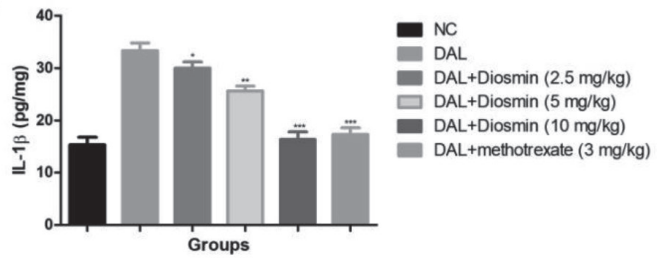

Fig. 8 The effect of diosmin on the pro-inflammatory cytokines on DAL induced solid tumor. a: TNF- $\alpha$, b: IL-1 $\beta$ and c: IL-6. Dennett's statical method was used for scrutinize the statistically significance ${ }^{*} p<0.05,{ }^{* *} p<0.01$ and ${ }^{* * *} p<$ $0.001)$.

\section{Discussion}

Cancer is a dreadful disease and is a set of various diseases categorized via uncontrolled cellular expansion, distant metastases and local tissue invasion ${ }^{20,21)}$. During the cancer disease, continuous generation of free radicals implicated the carcinogenesis and boosts the action of carcinogenesis $^{22,23)}$. Previous research suggests that the carcinogenesis is chronic, complicated and multistep process ${ }^{4,24)}$. The carcinogenesis enhanced the production of atypical cells leads to alteration in the gene expression factors predisposing it to malignancy which includes environmental agents, inherited traits, diet and cancer risk enhance with age. Studies suggest that the regular intake of plant based phyto-constituent reduce the effect of oxidative DNA injury in aged patient and also reduce the risk of cancer ${ }^{25)}$. Recently, investigation suggest that the plant based phytoconstituent especially flavonoids regular intake reduced the cancer and its related complication in the rodent model.

The valuable criterion to scrutinize the anti-cancer effect is prolongation of life span of rodent ${ }^{5)}$. During the expansion of cancer, reduce the life span due to expend the cancer into different tissue and organs. A similar result was observed in the solid tumor mice induced by DAL, showing the reduced lifespan, and diosmin significantly increased the dose-dependent lifespan, and suggesting the anticancer effect of the drugs tested. Body weight decreased during the enhance the ascites tumor volume. In the first 2 weeks after the DAL treatment, the ascitic fluid collection was quick in untreated mice, showing active secretion and deposition of ascitic fluid in the abdomen due to excess tumor expansion. DAL caused solid tumor mice showed the reduce body weight as compared to without treated group mice and diosmin significantly $(p<0.001)$ up-regulated the body weight as comparison to DAL bearing mice. Diosmin considerably decrease the tumor ascitic volume and clearly presents the potential effect of diosmin to slow down the tumor growth.

During the cancer, the very common feature is alteration of hematological parameters due to expend the disease into the circulation ${ }^{5)}$. Anomalous blood profile has been linked with the cancer ${ }^{7)}$. In the present experimental study, DAL induced solid tumor mice showed the augmented level of WBC, polymorphs and reduced level of hemoglobin, RBC. Our result clearly exhibited that diosmin increase the life span along with reduce the polymorphs and WBC at dose dependently. The common disadvantage during the chemotherapy is anemia and myelo-suppression. Anemia is the common issue during the cancer ${ }^{7)}$. In the current experimental study, DAL induced solid tumor mice showed the reduced level of $\mathrm{RBC}$ and hemoglobin. The most common side effect of cancer chemotherapy is the destruction of RBC may be due to deficiency of iron ${ }^{26)}$. Decreased the level of hemoglobin due to destruction of RBC. During the solid tumor progression, number of atypical leukocyte production is enhanced and number of peripheral count of WBC increased ${ }^{26)}$. DAL induced solid tumor mice showed the more anemic conditions due to suppression the hemoglobin and RBC level, which lead to myelopathic disease and iron deficiency.

During the alteration of blood profile, increased the level of cholesterol and reduced level of triglyceride was observed and confirm the Hodgkin lymphoma ${ }^{5)}$. Hodgkin lymphoma patients showed the alteration of liver enzymes due to increased the solid tumor. Hepatic parameters such as SGOT, SGPT and ALP are the common parameters. SGPT is the main enzymes derived from the hepatic tissue, bone and also found in the less amount in the kidneys, leucocytes, placenta and intestine ${ }^{5)}$. For hepatitis, cancer, and myocardial infarction the improved level of SGOT and SGPT was frequently observed. Furthermore, in the obstructive disease of the liver and biliary tract the level of 
hepatic enzyme such as ALP was boosted ${ }^{7)}$. ALP made-up of set $f$ enzymes that catalyzed the hydrolysis of phosphate esters in the alkaline environment, start the production of inorganic phosphate and organic radical ${ }^{7)}$. Significantly increased the serum level of ALP, is predominantly induces the malignant biliary cirrhosis, sarcoidosis and hepatic lymphoma. In the current experimental study, increased the level of hepatic parameters such as SGOT, SGPT and ALP in the DAL control mice. Diosmin and methotrexate diminished the SGOT, SGPT and ALP level and suggesting the chemoprotective effect. Diosmin reverse the increase level of hepatic enzymes level almost near to the normal value of mice.

It is well known that oxidative stress is consider as the cancer mediator. Reactive oxygen species (ROS) like hydrogen peroxides and organic have been play a significant role as tumor promoters ${ }^{27-29)}$. The biggest problem to enhance the disease is continuous generation of free radical, which induce the oxidative stress in the cell and tissues. Antioxidant have been exhibited to serve as subcellular messengers of normal cell function and also showed the protective role against the oxidative injury ${ }^{30)}$. Therefore, for searching the anticancer agents possessing the antioxidant effect is worthy in term of combined benefits of reduction of proliferation and scavenge the free radicals, toxicant inducing the oxidative stress in cancer. In this experimental study, we found the boosted level of TBARS (a LPO mediator) and its well proved that enhance level of TBARS boost the peroxidation chain. Diosmin considerably decreased the content of TBARS and suggest the reduction of peroxidation chain. The defense against endogenous antioxidants is an important part of the reduction of free radicals and oxidative stress ${ }^{19,31)}$. Endogenous antioxidant enzymes like SOD and CAT in the first line play an important role in inhibiting free radical production. SOD and CAT maintain the functional equilibrium between the pro-oxidant and antioxidant pathways ${ }^{31,32)}$. Enzymes like SOD and CAT, play an important role in defending the tissue against lipid peroxidation $^{26,33)}$. DAL induced solid tumor mice showed reduced endogenous enzyme levels (SOD and CAT) and diosmin significantly $(p<0.001)$ increased the SOD and CAT levels and closely near normal control. The harmful effect of free radicals is preserved through the cell defense antioxidant mechanism. The GSH is considering as the most crucial free radical chain breaking molecules. Previous research indicates that nonprotein thiol GSH is important for the proliferation and metabolism of tumor cells ${ }^{26,34)}$. The boosted rate of GSH is a tumor cell with tumor growth that may be involved in metabolism and tumor cell proliferation of the host for 14 days, for example, since GSH has been reported to sustain tumor cell proliferation through intracellular $\mathrm{pH}$ and regulate protein kinase activity C. DAL induced solid tumor mice showed the reduced concentration of GSH and diosmin and methotrexate increased the concentration of cellular GSH and suggesting the antioxidant effects.

Another approach for reducing the lipid peroxidation is enhancing the vitamin $\mathrm{C}$ and $\mathrm{E}$ level. Vitamin $\mathrm{C}$ is significant antioxidant parameter; it helps to restore the vitamin $\mathrm{E}$ level during the cancer disease condition ${ }^{26)}$. Another vitamin such as $\mathrm{E}$, is reacting via altering the cyclic process of lipid peroxidation reacting with superoxide radicals, before it can attack the lipid molecule. Diosmin substantially $(p<0.001)$ increased the level of vitamin $\mathrm{C}$ and decreased vitamin $\mathrm{E}$ levels and suggested chemoprotection.

Previous research suggests that the immune cells have an impact on the tumor growth, progression and initiation and many of these effects regulated via the pro-inflammatory cytokines ${ }^{26)}$. Pro-inflammatory cytokines such as IL-1 $\beta$, TNF- $\alpha$ and IL- 6 play an important role in the development of tumors. TNF- $\alpha$ (NF- $\alpha$ activator) plays an important role in inflammatory reaction growth and causes cell

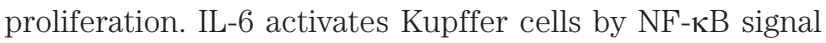
path activation. During the normal process, IL-6 protects the hepatic tissue damage from the disease ${ }^{26,35)}$. Another pro-inflammatory cytokines including IL-1 $\beta$ play a key role in inflammatory reaction expansion. Previous research suggests that the IL- $1 \beta$ induce the inflammatory reaction and increase level induce the chronic inflammation ${ }^{35,36)}$. DAL induced solid tumor mice showed the increased level of cytokines and diosmin significantly $(p<0.001)$ down-regulated cytokines and the level cytokines reach almost near to control range and suggesting the anti-inflammatory effect.

\section{Conclusion}

We can conclude that based on the results that DAL induced mice showed the increased level of hepatic, biochemical and antioxidant parameters and diosmin significantly reduced the biochemical, hepatic and antioxidant parameters. Diosmin increases the mice life span and reduces the tumor burden in the solid tumor mice caused by DAL. Diosmin significantly reduced the pro-inflammatory cytokines and suggest the anti-inflammatory effect. So diosmin may be further used to treat the rodent model of lymphoma and further detailed studies need to explore the possible mechanism of action.

\section{References}

1) Ferlay, J.; Soerjomataram, I.; Dikshit, R. et al. Cancer incidence and mortality worldwide: Sources, methods and major patterns in GLOBOCAN 2012. Int. J. Cancer 136, E359-E386 (2015).

2) Nasim, F.; Sabath, B.F.; Eapen, G.A. Lung cancer. Med. 
Clin. North Am. 103, 463-473(2019).

3) Velli, S.K; Sundaram, J; Murugan, M; Balaraman, G; Thiruvengadam, D. Protective effect of vanillic acid against benzo (a) pyrene induced lung cancer in Swiss albino mice. Journal of Biochemical and Molecular Toxicology 33, e22382(2019).

4) Nadeem, M.S; Kumar, V; Al-Abbasi, F.A; Kamal, M.A; Anwar, F. Risk of colorectal cancer in inflammatory bowel diseases. Semin. Cancer Biol. 64, 51-60 (2019).

5) Christina, A.J.M; Joseph, D.G; Packialakshmi, M. et al. Anticarcinogenic activity of Withania somnifera Dunal against Dalton's Ascitic Lymphoma. J. Ethnopharmacol. 93, 359-361 (2004).

6) Prasad, R; Koch, B. Antitumor activity of ethanolic extract of Dendrobium formosum in T-cell lymphoma: An in vitro and in vivo study. Biomed. Res. Int. 2014, 753451 (2014).

7) Chockalingam, V; Suryakiran Kadali, S.D.V; Gnanasambantham, P. Antiproliferative and antioxidant activity of Aegle marmelos (Linn.) leaves in Dalton's lymphoma ascites transplanted mice. Indian J. Pharmacol. 44, 225-229 (2012).

8) Li, C; Du, G.H. Diosmin. in Natural Small Molecule Drugs from Plants. Springer, pp. 65-69 (2018).

9) Tanaka, T; Makita, H; Kawabata, K. et al. Chemoprevention of azoxymethane-induced rat colon carcinogenesis by the naturally occurring flavonoids, diosmin and hesperidin. Carcinogenesis 18, 957-965(1997).

10) Dholakiya, S.L; Benzeroual, K.E. Protective effect of diosmin on LPS-induced apoptosis in PC12 cells and inhibition of TNF- $\alpha$ expression. Toxicol. Vitr. 25, 1039-1044(2011).

11) Tahir, M; Rehman, M.U; Lateef, A. et al. Diosmin protects against ethanol-induced hepatic injury via alleviation of inflammation and regulation of TNF- $\alpha$ and NF-кB activation. Alcohol. 47, 131-139(2013).

12) Sawmiller, D; Habib, A; Li, S. et al. Diosmin reduces cerebral A $\beta$ levels, tau hyperphosphorylation, neuroinflammation, and cognitive impairment in the $3 \mathrm{xTg}-\mathrm{AD}$ mice. J. Neuroimmunol. 299, 98-106 (2016).

13) Imam, F; Al-Harbi, N.O; Al-Harbi, M.M. et al. Diosmin downregulates the expression of $\mathrm{T}$ cell receptors, proinflammatory cytokines and NF- $\mathrm{NB}$ activation against LPS-induced acute lung injury in mice. Pharmacol. Res. 102, 1-11 (2015).

14) Pari, L; Srinivasan, S. Antihyperglycemic effect of diosmin on hepatic key enzymes of carbohydrate metabolism in streptozotocin-nicotinamide-induced diabetic rats. Biomed. Pharmacother. 64, 477-481 (2010).

15) Antony, J.J; Sithika, M.A.A; Joseph, T.A. et al. In vivo antitumor activity of biosynthesized silver nanoparticles using Ficus religiosa as a nanofactory in DAL induced mice model. Colloids Surf. B 108, 185-190
(2013).

16) Sriram, M.I; Kanth, S.B.M; Kalishwaralal, K; Gurunathan, S. Antitumor activity of silver nanoparticles in Dalton's lymphoma ascites tumor model. Int. J. Nanomedicine 5, 753-762 (2010).

17) Sunila, E.S; Kuttan, G. Immunomodulatory and antitumor activity of Piper longum Linn. and piperine. J. Ethnopharmacol. 90, 339-346(2004).

18) Sreejamole, K.L; Radhakrishnan, C.K. Antioxidant and cytotoxic activities of ethyl acetate extract of the Indian green mussel Perna viridis. Asian J. Pharm. Clin. Res. 6, 197-201 (2013).

19) Dhamija, I; Kumar, N; Manjula, S.N; Parihar, V; Setty, M.M; Pai, K.S.R. Preliminary evaluation of in vitro cytotoxicity and in vivo antitumor activity of Premna herbacea Roxb. in Ehrlich ascites carcinoma model and Dalton's lymphoma ascites model. Exp. Toxicol. Pathol. 65, 235-242 (2013).

20) Harrington, K.J. The biology of cancer. Med (United Kingdom). 44(1), 1-5(2016).

21) Stewart, B.W; Wild, C.P. World cancer report 2014. World Heal Organ 7 (2), 418-419 (2014).

22) Birben, E; Sahiner, U.M; Sackesen, C; Erzurum, S; Kalayci, O. Oxidative stress and antioxidant defense. World Allergy Organ J. 5, 9-19(2012).

23) Sun, J; Chu, Y.F; Wu, X; Liu, R.H. Antioxidant and antiproliferative activities of common fruits. J. Agric. Food Chem. 44, 701-705(2002).

24) Yuan, C; Wang, C; Wang, J. et al. Inhibition on the growth of human MDA-MB-231 breast cancer cells in vitro and tumor growth in a mouse xenograft model by Se-containing polysaccharides from Pyracantha fortuneana. Nutr. Res. 36, 1243-1254(2016).

25) Saha, S; Mukhopadhyay, M.K; Ghosh, P.D; Nath, D. Effect of methanolic leaf extract of Ocimum basilicum L. on benzene-induced hematotoxicity in mice. Evid. Based Complement. Altern. Med. 2012, 176385 (2012).

26) Rubila, S; Ranganathan, T.V; Sakthivel, K.M. Protective effect of Zingiber officinale against Dalton's Lymphoma Ascites Tumour by regulating inflammatory mediator and cytokines. Appl. Biochem. Biotechnol. 180, 1482-1496 (2016).

27) Kumar, V; Ahmed, D; Gupta, P.S; Anwar, F; Mujeeb, M. Anti-diabetic, anti-oxidant and anti-hyperlipidemic activities of Melastoma malabathricum Linn. leaves in streptozotocin induced diabetic rats. BMC Complement. Altern. Med. 13, 222 (2013).

28) Kumar, V; Ahmed, D; Verma, A; Anwar, F; Ali, M; Mujeeb, M. Umbelliferone $\beta$-D-galactopyranoside from Aegle marmelos (L.) corr. An ethnomedicinal plant with antidiabetic, antihyperlipidemic and antioxidative activity. BMC Complement. Altern. Med. 13, 273 (2013). 
29) Kumar, V; Bhatt, P.C; Rahman, M. et al. Melastoma malabathricum Linn attenuates complete freund's adjuvant-induced chronic inflammation in Wistar rats via inflammation response. BMC Complement. Altern. Med. 16, 510 (2016).

30) Merlin, N.J; Parthasarathy, V. Potential antitumour activity of Gmelina asiatica aerial parts against dalton ascites lymphoma in mice. Asian J. Chem. 22, 31933199 (2010).

31) Balamurugan, K; Nishanthini, A; Mohan, V.R. Anticancer activity of ethanol extract of Melastoma malabathricum L. Leaf against dalton ascites lymphoma. J. Pharm. Sci. Res. 5(5), 111-114(2013).

32) Augustine, B.B; Dash, S; Lahkar, M; Sarma, U; Samudrala, P.K; Thomas, J.M. Leucas aspera inhibits the Dalton's ascitic lymphoma in Swiss albino mice: A preliminary study exploring possible mechanism of action. Pharmacogn. Mag. 10(38), 118-124(2014).
33) Thavamani, B.S; Mathew, M; Palaniswamy, D.S. Anticancer activity of Cocculus hirsutus against Dalton's lymphoma ascites (DLA) cells in mice. Pharm. Biol. 52, 867-872 (2014).

34) Sivakumar, P; Sambath Kumar, R; Sivakumar, T. et al. Antitumor and antioxidant activities of Triumfetta rhomboidea against Dalton's ascites lymphoma bearing Swiss albino mice. Res. J. Med. Sci. 1, 486-494 (2008).

35) Khynriam, D; Prasad, S.B. Hematotoxicity and blood glutathione levels after cisplatin treatment of tumorbearing mice. Cell Biol. Toxicol. 17, 357-370 (2001).

36) Shanmugapriya, K; Peer Mohammed, S; Saravana, P.S. Evaluation of phytochemical constituents and pharmacological activities of ethanolic extract of Piper nigrum leaves against DLA in Swiss albino mice. Int. $J$. Pharmacogn. Phytochem. Res. 6, 27-38(2014). 